\title{
BMJ Open Light-moderate alcohol consumption and left ventricular function among healthy, middle-aged adults: the HUNT study
}

\author{
Katalin Gémes, ${ }^{1}$ Imre Janszky, ${ }^{2,3}$ Linn Beate Strand, ${ }^{2}$ Krisztina D László, ${ }^{1}$ \\ Staffan Ahnve, ${ }^{1}$ Lars J Vatten, ${ }^{2}$ Håvard Dalen, ${ }^{4,5,6}$ Kenneth J Mukamal ${ }^{7}$
}

To cite: Gémes $\mathrm{K}$, Janszky I, Strand LB, et al. Light-moderate alcohol consumption and left ventricular function among healthy, middle-aged adults: the HUNT study. BMJ Open 2018;8:e020777. doi:10.1136/ bmjopen-2017-020777

- Prepublication history and additional material for this paper are available online. To view these files, please visit the journal online (http://dx.doi. org/10.1136/bmjopen-2017020777).

Received 26 November 2017 Revised 12 January 2018 Accepted 1 February 2018

Check for updates

For numbered affiliations see end of article.

Correspondence to

Dr. Imre Janszky;

imre.janszky@ntnu.no

\section{ABSTRACT}

Objectives To investigate the association between alcohol consumption and left ventricular (LV) function in a population with low average alcohol intake.

Design, setting and participants A total of 1296 healthy participants, free from cardiovascular diseases, were randomly selected from the third wave of the Norwegian HUNT study (2006-2008) and underwent echocardiography. After validation of the inclusion criteria, 30 participants were excluded due to arrhythmias or myocardial or valvular pathology. Alcohol consumption, sociodemographic and major cardiovascular risk factors were assessed by questionnaires and clinical examination in the HUNT3. General linear models were used to analyse the cross-sectional associations between alcohol intake and LV indices.

Primary and secondary outcome measures LV functional and structural indices were measured with tissue Doppler and speckle tracking echocardiography. Results We observed no associations between alcohol consumption and multivariable-adjusted LV functional indices. Excluding abstainers who reported regular alcohol consumption 10 years prior to the baseline did not change the results. Alcohol consumption was positively associated with LV mass indices $(p<0.01$ for linear trend of the means); there was no such association among participants with non-risky drinking characteristics ( $p=0.67$ for linear trend of the means).

Conclusions We found no clear evidence that lightmoderate alcohol consumption is associated with measures of LV function, although our results indicate that consumption, especially when marked by binge drinking, is progressively associated with greater LV mass.

\section{INTRODUCTION}

While excessive alcohol consumption is clearly responsible for alcohol-related cardiomyopathy, ${ }^{12}$ several studies suggest that frequent but moderate alcohol consumption is associated with lower risk of heart failure. ${ }^{3}$ Some studies have examined the associations of light-moderate alcohol consumption with subclinical structural and functional properties of the left ventricle among asymptomatic

\section{Strengths and limitations of this study}

- The HUNT was conducted in a socioeconomically and genetically homogenous population, where due to the strict alcohol policy the majority of the population was non-drinkers or light drinkers which decreased confounding by social factors in this study. This, together with the statistical adjustment for a wide range of cardiovascular risk factors, reduces the possibility of residual confounding in our study.

- Alcohol consumption was also assessed 10 years prior to baseline as part of the HUNT2 study, offering a prospective assessment of former drinking.

- In this study, greyscale speckle tracking and tissue Doppler echocardiography was used, which provides more sensitive left ventricular functional indices than ejection fraction measures.

- The main limitation of our study relates to its cross-sectional design.

- As in many previous studies in this field, alcohol consumption was self-reported.

participants, but the results of these studies have been inconsistent. Although most describe increasing LV mass with increasing level of alcohol consumption, ${ }^{4-7}$ the associations with LV function are less consistent. Some found better LV function with increasing alcohol intake, ${ }^{4}$ while others reported the opposite. ${ }^{5}{ }^{6}$ Most studies assessed ejection fraction (EF), a potentially less sensitive index of $\mathrm{LV}$ functional impairment. ${ }^{89}$ Speckle tracking and tissue Doppler echocardiography may provide more direct measures of LV contractile function. ${ }^{8}$ 10-13 Furthermore, the majority of these studies have been subject to several potential limitations. A major methodological problem that limits causal inference in studies regarding the health effects of alcohol is the so called 'sick-quitter' bias. ${ }^{14} 15$ Thus the U-shaped or J-shaped association often found for alcohol consumption and many health outcomes ${ }^{14-16}$ 


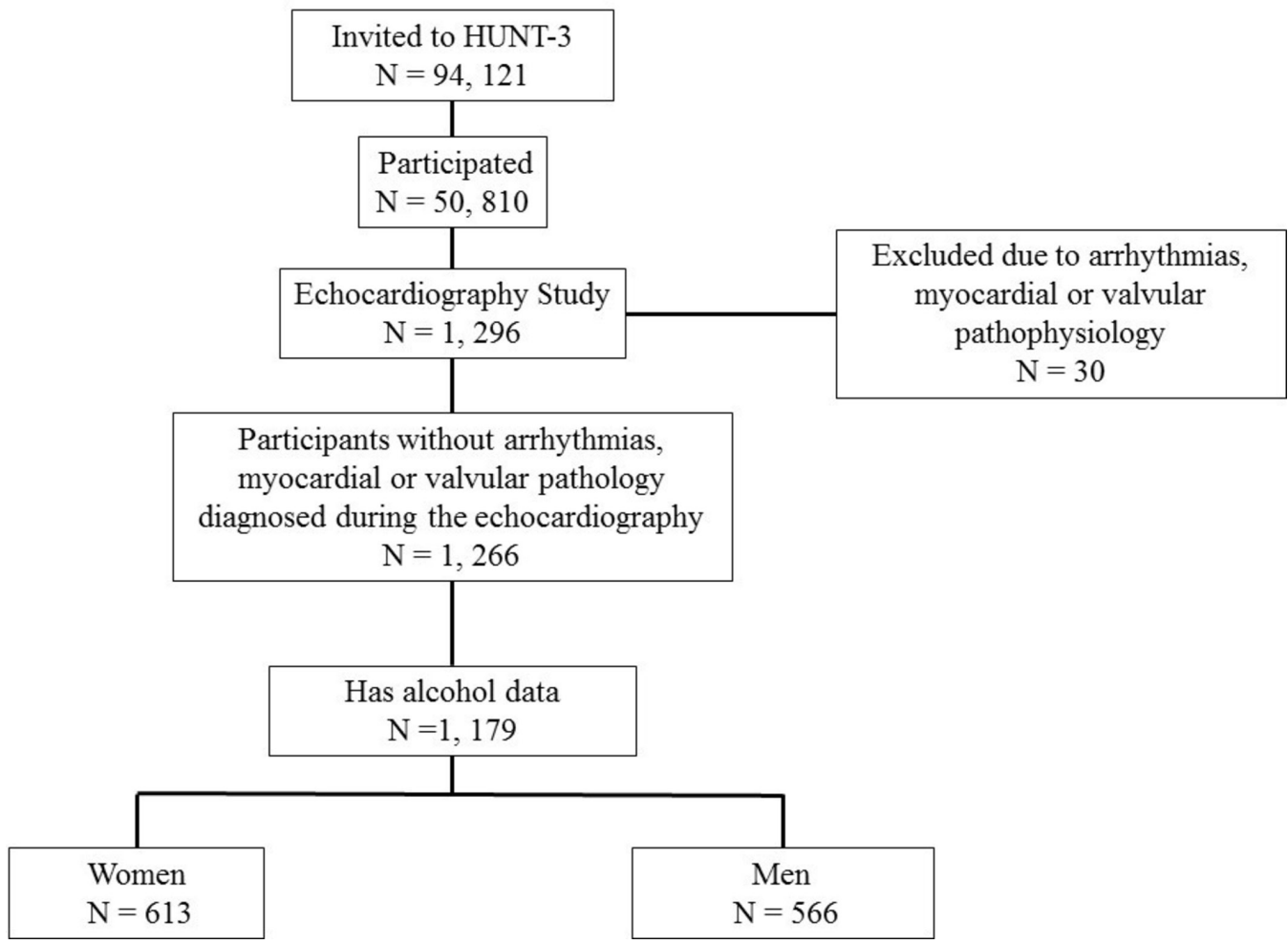

Figure 1 Flowchart for the selection of the study population.

might be an artefact caused by the higher risk of adverse health outcomes among abstainers who quit drinking due to their ill health. ${ }^{17}$

In addition, most previous studies only assessed the quantity of alcohol intake ${ }^{4-6}$ and not the drinking pattern (ie, heavy episodic vs regular, low amount of alcohol consumption) at identical quantities of intake, which might potentially modify the association between alcohol consumption and LV function. ${ }^{6}$

In this study, we examined the associations of alcohol intake with LV functional and structural measures in a healthy middle-aged population. We also assessed drinking pattern and quantity of alcohol consumption 10 years prior to baseline. To detect early, subclinical signs of myocardial dysfunction, LV functional indices were measured using highly sensitive tissue Doppler and speckle tracking echocardiography. ${ }^{13} 18$

\section{METHODS}

\section{Study population}

Between 2006 and 2008, the entire adult population of the Nord-Trøndelag County in Norway, representing 93210 citizens, was invited to participate in the third wave of Nord-Trøndelag Health Study (HUNT3) (http://www.ntnu.edu/hunt). Altogether, 50810 individuals (54\% of those invited) participated in the study. ${ }^{19}$ Data on sociodemographic factors, anthropometrics, overall health status, chronic health conditions and health behaviour were collected by self-reported questionnaires, interviews and clinical examinations. ${ }^{19}$
A subsample of healthy individuals without known cardiovascular diseases (CVDs), diabetes or hypertension ( $\mathrm{n}=1$ 296) was randomly selected from the HUNT3 cohort to participate in the Echocardiography Study. ${ }^{13} 18$ After validation of the inclusion criteria by an experienced physician echocardiographer, 30 participants were excluded from the study due to arrhythmias or myocardial or valvular pathology. ${ }^{13} 18$ In addition, for the analyses of the present study, we excluded 87 participants with missing data on alcohol consumption (figure 1).

The Echocardiography study was approved and it was conducted according to the second Helsinki Declaration.

\section{Measures}

Alcohol consumption

Alcohol consumption was assessed by self-administered questionnaires. Participants reported the amount and type of alcoholic beverages they consume in a usual 2 -week period. The frequency of alcohol consumption was assessed for the previous 12 months. Those who answered that they did not drink any alcohol in the previous 12 months were considered abstainers. Those who reported any alcohol consumption during the previous year but reported no consumption during a usual 2-week period were categorised as rare drinkers. Those who reported at least one standard drink during a usual 2-week period were categorised as having an intake of one of the following: 'at least 0.5 and less than or equal to three drinks/week', 'more than three and less than or equal to seven drinks/week' or 'more than seven drinks/week'. The frequency of drinking was categorised as: consuming 
alcoholic beverages 'less than once a month', '1-4 times a month' or 'more than once a week'. Binge drinking was defined as consuming more than five drinks in one sitting. Problem drinking behaviour was assessed by the CAGE questionnaire. ${ }^{20}$ Risky drinkers were defined as those who answered positively to at least two questions of the CAGE questionnaire ${ }^{21}$ and/or reported binge drinking at least once a month. Overall, $50 \%$ of the participants gave information about their alcohol consumption in the second wave of the HUNT Study (HUNT2) conducted approximately 10 years prior to HUNT3. ${ }^{19}$ Current non-drinkers were categorised as 'long-term abstainers' or 'former drinkers, but current abstainers' according to their previous alcohol intake.

\section{Echocardiography}

Echocardiograms were recorded in the left-lateral decubitus position by a Vivid 7 high-end scanner (V.BT06, GE Ultrasound, Horten, Norway) with a phased-array transducer (M3S and M4S) assessed by an experienced physician echocardiographer. Analyses were performed in EchoPAC SWO software (GE Ultrasound) if not otherwise specified. The detailed protocol, methodology and validity of the echocardiographic measurement have been published elsewhere. ${ }^{1318}$ Shortly, the mean errors of the LV function indices were 4\%-9\% (test- retest, two observers analysing separate recordings).

LV function was assessed by well-established echocardiographic indices of the diastolic and systolic longitudinal function. Global longitudinal end-systolic strain (percentage shortening of the left ventricle during systole) and peak global strain rate (the maximal speed of the global longitudinal strain) were measured as the average of the segmental values according to a 16-segment model of the left ventricle ${ }^{22}$ using a combined tissue Doppler and greyscale speckle tracking method in a customised software package (GcMat) based on a MatLab platform (MathWorks, Natick, Massachusetts, USA). ${ }^{13} 18$ Both LV longitudinal strain and velocity can detect subclinical myocardial dysfunction before EF is materially affected. These indices are also associated with acute myocardial infarction, heart failure and cardiovascular and all-cause mortality. ${ }^{10} 23$

Systolic mitral annular excursion (MAE) was measured by corrected motion mode from greyscale recordings as the average of the total systolic excursion of the mitral annular plane at the inferoseptal, lateral, anterior and inferior positions of the LV wall. Peak systolic (S') and peak early diastolic mitral annular velocity (e') were calculated as the average of peak velocities measured at the same locations by pulsed wave tissue Doppler echocardiography. ${ }^{13} 18$

Tricuspid annular plane systolic excursion (TAPSE) and tricuspid annular peak systolic velocity (RS') were measured close to the tricuspid plane in the free wall of the right ventricle, by similar methodologies as described for the MAE and mitral annular velocities.
Mitral inflow early (E) and late (A) diastolic velocities were recorded by pulse wave Doppler, and the E/A ratio was calculated. $\mathrm{E} / \mathrm{e}$ ' ratio was calculated as the ratio of the peak early diastolic mitral inflow per mitral annular early diastolic velocities.

Conventional LV structural indices (interventricular septum and posterior wall thickness and LV internal dimensions) were assessed in parasternal M-mode. LV mass was estimated according to the Cube formula and indexed for body surface area (BSA). Relative wall thickness was defined as 2xLV posterior wall thickness divided by the LV end-diastolic diameter.

\section{Sociodemographic and lifestyle factors}

Highest achieved education was categorised as primary or lower secondary school ( $<10$ years), upper secondary school (10-12 years) or high school/university (>12 years). Marital status was categorised as: (1) never married, (2) married or cohabiting and (3) separated or widowed.

Participants were classified as never, former or current smokers. A validated physical activity index was calculated based on the reported frequency, duration and intensity of the physical activity, as previously described. ${ }^{24}$

\section{Clinical examination}

Weight, height and blood pressure were measured by trained nurses. Body mass index (BMI) was calculated by dividing weight in kilogram by height ${ }^{2}$ in metres and dichotomised at $25 \mathrm{~kg} / \mathrm{m}^{2}$. BSA was calculated according to the DuBois formula. Information on common chronic disorders were self-reported by the participants (described in detail elsewhere). ${ }^{19}$

\section{Statistical analyses}

As the reference values for most of the echocardiographic measurements differ according to sex, we conducted our main analyses for the whole population and separately for men and women. ${ }^{22}{ }^{25}$ To examine the association between alcohol consumption and echocardiographic indices of cardiac structure and function, we performed general linear models. Least square means of cardiac function indices (MAE, global longitudinal strain, global longitudinal strain rate, peak early diastolic and systolic mitral annular velocities (e', S'), E/e', E/A, TAPSE and RS') and LV structural indices (myocardial mass, wall thickness and dimensions) and $95 \%$ CIs were calculated across alcohol consumption categories. In light of covariates, we conducted full-case analyses. We adjusted our analyses for age, education, marital status, physical activity, smoking, BMI and sex (if not stratified). In additional analyses, we further included systolic blood pressure, which may be a consequence of alcohol consumption. We tested linear as well as quadratic trends. As estimates did not differ between the abstainer and rare-drinker groups, we combined these two groups (as non-drinkers) for further analyses and present all results accordingly. 
To test for the 'sick quitters' bias, ${ }^{14} 15$ we repeated our main analyses after excluding abstainers who reported alcohol intake during the earlier HUNT2. To examine whether risky drinking modified the observed associations, we performed stratified analyses by reported binge and/or problem drinking and tested interaction between alcohol intake and binge and/or problem drinking using Wald tests. We also added the frequency of alcohol intake to model 2 while simultaneously adjusting for the amount of alcohol consumption to determine the relative contributions of quantity and frequency of intake. Beverage specific analyses were conducted in the same fashion. To investigate the effect of a specific type of beverage, for example, wine, we examined the association between alcohol intake and LV functional and structural indices while adjusting for amounts consumed of the other two beverages, that is, beer and spirit in this example.

To assess effect modification, we stratified our analyses by sex, age (dichotomised at 50 years), smoking and BMI (dichotomised at $25 \mathrm{~kg} / \mathrm{m}^{2}$ ) and also tested for the interaction between alcohol consumption and the effect modifiers. As previous studies usually assessed LV function as EF, we also performed sensitivity analyses with this outcome to improve the comparability of our results with those of previous studies. In other sensitivity analysis, we examined age-adjusted models using alcohol consumption from HUNT2 as exposure and MAE, strain, strain rate, S' and e' and LV mass from HUNT3 as the outcome.

Statistical analyses were performed using SAS Enterprise Guide V.6.0 (SAS Institute) and Stata IC/12.1 for Windows (Stata).

\section{RESULTS}

Table 1 shows the characteristics of study participants separately for men and women. The majority of both men and women were light drinkers, consuming less than three alcoholic drinks per week; approximately $4.5 \%$ of both men and women reported consumption of more than seven alcoholic drinks per week. For both genders, drinkers were more likely to be smokers and to have a slightly lower BMI than non-drinkers. Men who reported alcohol consumption tended to be younger than abstainers.

\section{Amount of alcohol consumption}

Table 2a and b shows the multivariable-adjusted means of LV indices, which were largely similar across alcohol intake categories. Quantity of alcohol intake was associated with higher LV mass, indexed LV mass and LV end-diastolic dimension in both men and women. The results were essentially the same when systolic blood pressure was added to the models. Excluding former drinkers $(\mathrm{n}=117)$ did not influence the association. There was no meaningful association between alcohol intake and EF (online supplementary eTable 1).

\section{Drinking pattern and beverage type analyses}

In general, drinking frequency did not influence the associations between alcohol intake and LV indices (online supplementary eTable 2). When we stratified our analyses according to risky and non-risky drinking, we found a weak tendency toward higher values on some of the LV indices with higher alcohol intake among participants without risky drinking, and a linear increase in LV mass indices among risky drinkers (table 3). Risky drinking was positively associated with $\mathrm{LV}$ mass $(\mathrm{P}<0.01)$ but not with the $\mathrm{LV}$ functional indices, independently from the amount of alcohol intake. The associations between alcohol consumption and LV indices did not differ according to beverage type nor did we find any consistent evidence for effect modification by age, sex, BMI or smoking status. We found no clear association between alcohol consumption assessed in HUNT2 and LV functional indices and LV mass in HUNT3, but the precision of our estimates was limited by the 573 individuals from our sample participated in both HUNT2 and 3 (see online supplementary eTable 3 ).

\section{DISCUSSION}

We found no clear evidence for a clinically meaningful association between light-moderate alcohol consumption and several sensitive echocardiographic indices for assessment of systolic and diastolic left and right ventricle function in this cohort of healthy individuals. However, LV mass and LV end-diastolic diameter showed positive linear associations with alcohol consumption, and the association with LV mass was particularly strong among participants with a risky drinking pattern. Neither frequency of drinking nor beverage types showed clear associations with the studied echocardiographic indices beyond the quantity of alcohol consumed.

\section{Comparison with previous studies}

Our study adds to a still limited literature relating alcohol consumption in the general population and LV function indices. In the present study, LV function was assessed by highly sensitive echocardiographic methods ${ }^{1318}$ in a healthy middle-aged Caucasian population. Previous studies on alcohol consumption and LV function sometimes used less sensitive measurements on LV function such as ejection fraction, ${ }^{46}$ while others were restricted to elderly ${ }^{5}$ or Asian participants. ${ }^{6}$ Among the most sensitive indices used to reveal LV diastolic and systolic dysfunction are global longitudinal strain and the peak systolic and early diastolic mitral annular velocities ( $\mathrm{S}^{\prime}$ and $\left.\mathrm{e}^{\prime}\right)$. The indices are sensitive to LV subclinical dysfunction and give prognostic information related to heart failure, cardiovascular death and risk of myocardial infarction. ${ }^{23}{ }^{27}$ Goncalves $e t a \tilde{l}$ found a positive association between alcohol intake and peak early diastolic mitral annular velocity (e') in men. ${ }^{5}$ We could not confirm such an association, but e' was slightly higher among women, who reported drinking three to seven alcoholic drinks per week, compared with women who reported less than this amount. On the other hand, alcohol intake 


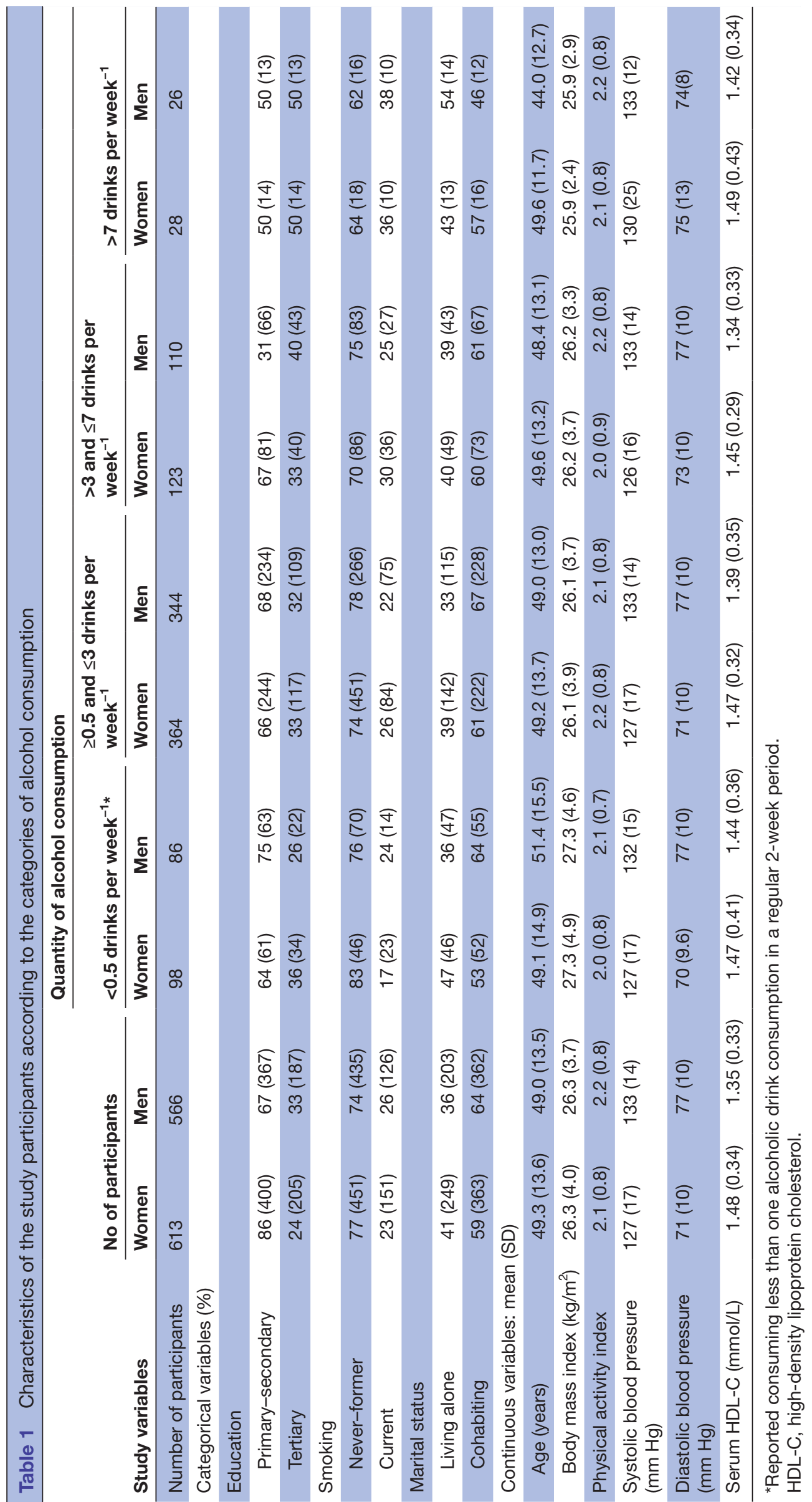




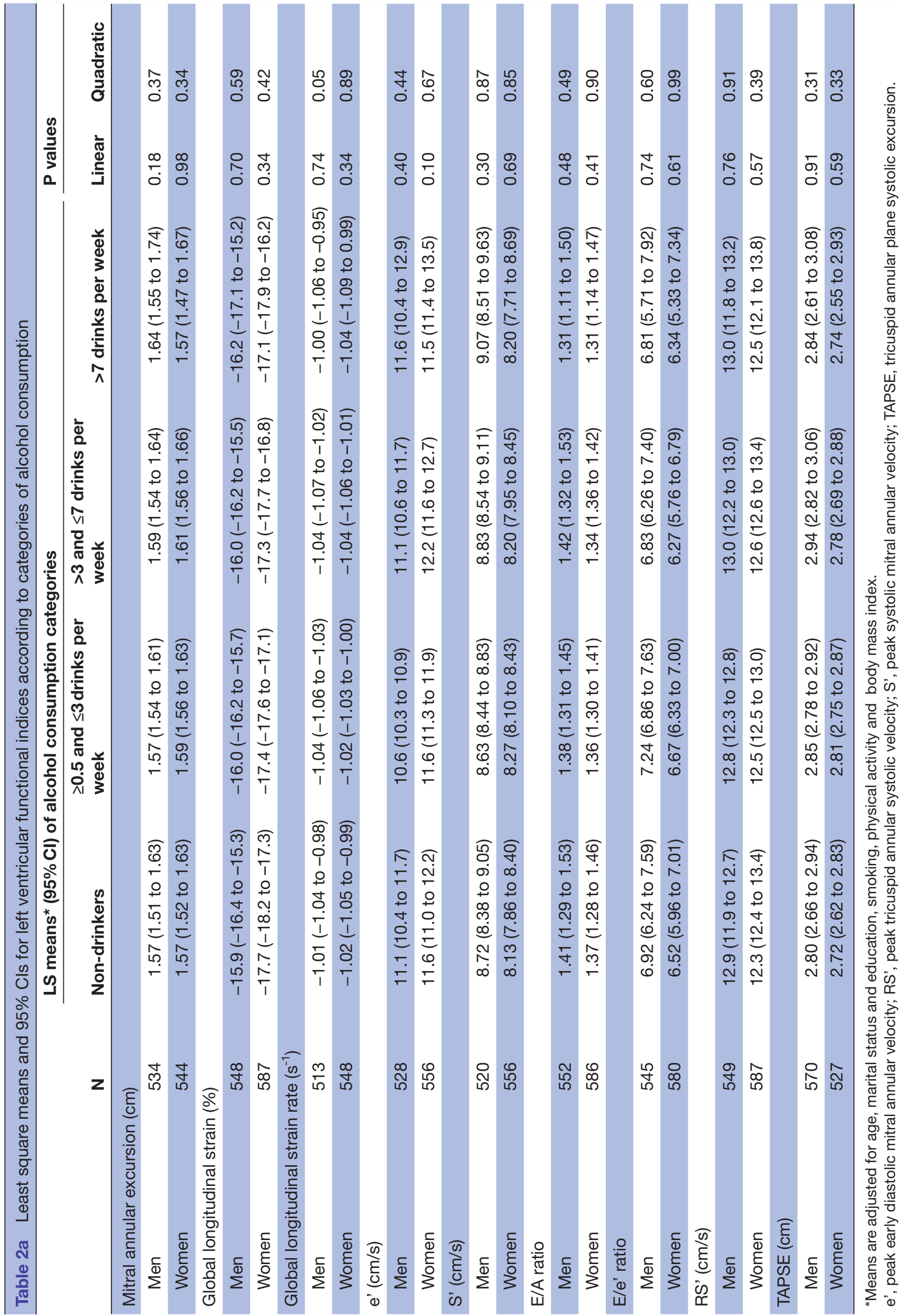

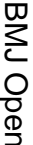

ज

응

$\frac{\bar{\sigma}}{0}$

क

$\overrightarrow{\vec{\omega}}$ 


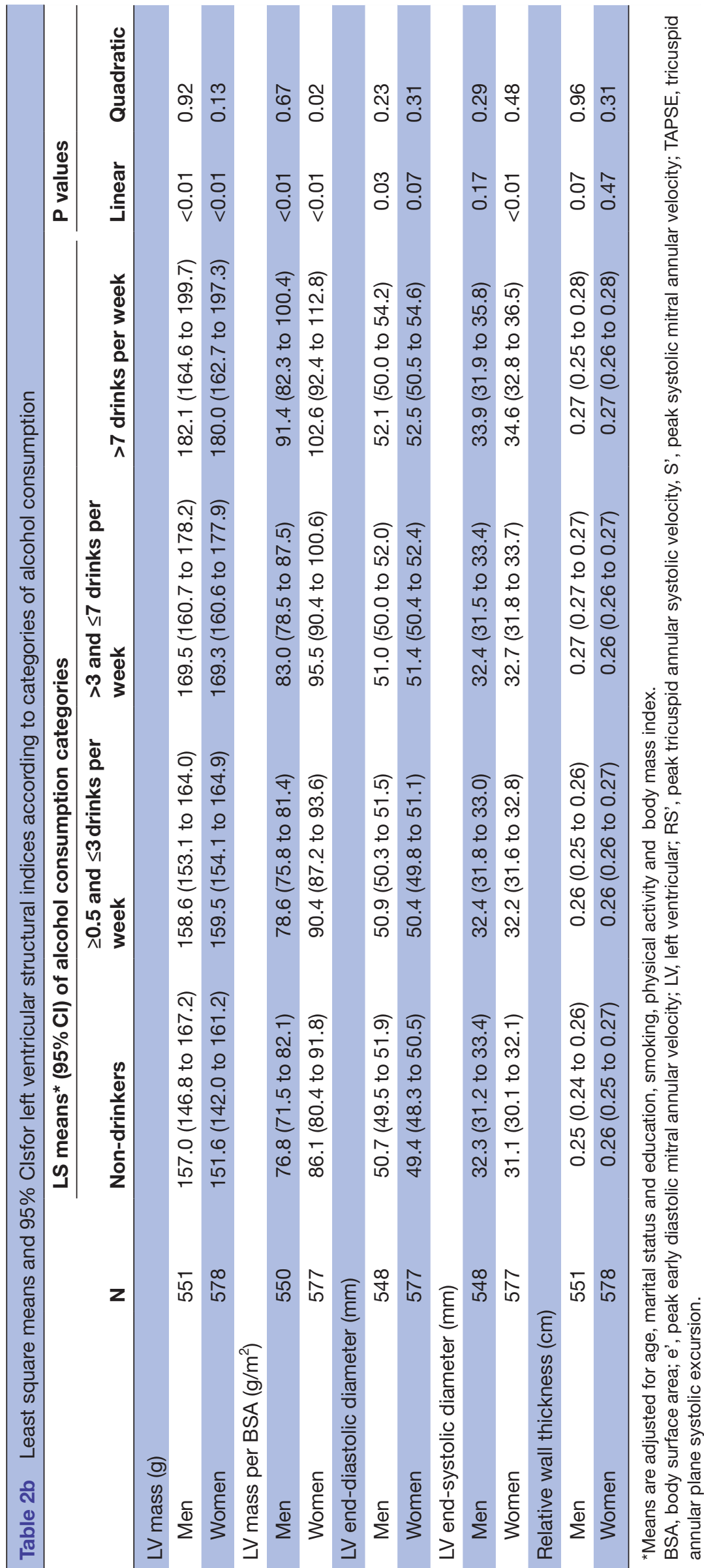


Table 3 Least square means and $95 \%$ of $\mathrm{Cl}$ for the left ventricular function indices according to problem and/or binge drinking and quantity of alcohol consumption

\begin{tabular}{|c|c|c|c|c|}
\hline \multirow[b]{2}{*}{ LV functional and structural indices } & \multicolumn{2}{|c|}{$\begin{array}{l}\text { Participants without problem and/or binge } \\
\text { drinking }\end{array}$} & \multicolumn{2}{|c|}{$\begin{array}{l}\text { Participants with problem and/orbinge } \\
\text { drinking }\end{array}$} \\
\hline & Mean* & $95 \% \mathrm{Cl}$ & Mean* & $95 \% \mathrm{Cl}$ \\
\hline Non-drinker & 1.60 & 1.46 to 1.73 & 1.60 & 1.46 to 1.73 \\
\hline$\geq 0.5$ and $\leq 3$ drinks/week & 1.61 & 1.57 to 1.64 & 1.61 & 1.57 to 1.64 \\
\hline$>3$ and $\leq 7$ drinks/week & 1.62 & 1.58 to 1.67 & 1.62 & 1.58 to 1.67 \\
\hline$P$ values (linear) & 0.97 & & 0.85 & \\
\hline$P$ values (quadratic) & 0.87 & & 0.78 & \\
\hline Global longitudinal strain (\%) & $\mathrm{n}=735$ & & $n=404$ & \\
\hline Non-drinker & -16.6 & -17.3 to -16.0 & -17.3 & -18.6 to 16.0 \\
\hline$P$ values (quadratic) & 0.839 & & 0.59 & \\
\hline Global longitudinal strain rate (/s) & $n=727$ & & $n=399$ & \\
\hline Non-drinker & -0.99 & -1.02 to -0.95 & -1.03 & -1.11 to 0.95 \\
\hline$\geq 0.5$ and $\leq 3$ drinks/week & -1.03 & -1.04 to -1.02 & -1.04 & -1.06 to 1.02 \\
\hline$>3$ and $\leq 7$ drinks/week & -1.04 & -1.07 to -1.01 & -1.05 & -1.07 to 1.02 \\
\hline$>7$ drinks/week & -1.02 & -1.10 to -0.95 & -1.02 & -1.06 to 0.97 \\
\hline$P$ values (linear) & 0.348 & & 0.83 & \\
\hline$P$ values (quadratic) & 0.166 & & 0.39 & \\
\hline$e^{\prime}(\mathrm{cm} / \mathrm{s})$ & $n=580$ & & $n=403$ & \\
\hline$P$ values (quadratic) & 0.37 & & 0.56 & \\
\hline $\mathrm{S}^{\prime}(\mathrm{cm} / \mathrm{s})$ & $\mathrm{n}=580$ & & $n=403$ & \\
\hline Non-drinker & 8.49 & 8.14 to 8.85 & 8.16 & 7.41 to 8.91 \\
\hline$\geq 0.5$ and $\leq 3$ drinks/week & 8.39 & 8.26 to 8.52 & 8.43 & 8.25 to 8.62 \\
\hline$>3$ and $\leq 7$ drinks/week & 8.54 & 8.25 to 8.82 & 8.42 & 8.18 to 8.65 \\
\hline$>7$ drinks/week & 9.32 & 9.32 to 10.09 & 8.38 & 7.96 to 8.81 \\
\hline $\mathrm{P}$ values (linear) & 0.05 & & 0.63 & \\
\hline$P$ values (quadratic) & 0.05 & & 0.52 & \\
\hline LV mass per BSA $\left(\mathrm{gr} / \mathrm{m}^{2}\right) \dagger$ & $\mathrm{n}=571$ & & $n=403$ & \\
\hline Non-drinker & 83.6 & 76.9 to 90.4 & 87.8 & 73.7 to 101.8 \\
\hline$\geq 0.5$ and $\leq 3$ drinks/week & 83.7 & 81.3 to 86.2 & 88.8 & 85.5 to 92.1 \\
\hline$>3$ and $\leq 7$ drinks/week & 82.7 & 77.4 to 88.1 & 93.4 & 89.2 to 97.6 \\
\hline$>7$ drinks/week & 80.4 & 66.1 to 94.7 & 100.88 & 93.3 to 108.4 \\
\hline $\mathrm{P}$ values (linear) & 0.67 & & 0.07 & \\
\hline$P$ values (quadratic) & 0.78 & & 0.46 & \\
\hline
\end{tabular}

Problem drinking was defined as having at least two positive answers on the CAGE questionnaire or reporting more than one binge drinking occasion during the last month.

${ }^{*}$ Adjusted for age, marital status, education, smoking, body mass index and physical activity.

†The $P$ values for the test for interaction was $<0.05$.

BSA, body surface area; e', peak early diastolic mitral annular velocity; LV, left ventricular; S', peak systolic mitral annular velocity. 
(especially in men) showed a positive but weak association with peak systolic mitral annular velocity ( $\left.\mathrm{S}^{\prime}\right)$. However, consistent with previous studies, ${ }^{56}$ we did not find clinically meaningful differences in most indices between the different alcohol intake categories.

Concerning structural characteristics, both our study and the study by Goncalves and colleagues ${ }^{5}$ found marginally higher LV end-diastolic and end-systolic dimensions in higher alcohol consumption categories. We also found a positive association between the quantity of alcohol consumption and LV mass, an established risk factor for CVD. ${ }^{28}$ Previous studies have also shown increased LV mass, but only in individuals with alcohol consumption above 14 drinks per week. ${ }^{45} 729$ However, our results provide important information to the relationship between alcohol consumption and structural cardiac changes by, to our knowledge, including for the first time information on risky drinking.

Heavy episodic drinking can lead to myocardial damage ${ }^{13031}$ and can be responsible for impaired LV function. ${ }^{2}$ It is associated with higher risk for CVD, even if the overall alcohol consumption stays at a moderate level. ${ }^{16}$ 32-34 Indeed, one binge-drinking episode (ie, consuming more than five drinks in one setting during a short period of time) can cause a pronounced inflammatory response in the myocardium. ${ }^{35}$ In our study, we found a clear increase in LV mass with higher reported alcohol intake among individuals with risky drinking patterns, but no such association among those who denied bingeing and problem drinking. The finding of higher LV mass among individuals with risky drinking patterns might be a subtle sign of ongoing cardiac remodelling, without any sign of or preceding subclinical dysfunction. This finding also fits well with the established effects of binge drinking (or 'holiday heart') on atrial arrhythmias. ${ }^{36}$

\section{Strengths and limitations}

The HUNT was conducted in a socioeconomically and genetically homogenous population. ${ }^{19}$ Furthermore, due to a relatively strict Norwegian alcohol policy, which includes high tax, limited availability of alcoholic beverages only in state-owned alcohol shops and promotion of alcohol-free public places, non-drinking is culturally more accepted than in most other Western countries, ${ }^{37}$ where moderate drinking is the cultural norm and both abstainers and heavy drinkers are often socially less active individuals with less social support and more psychological distress. ${ }^{38} 39$

This, together with the statistical adjustment for a wide range of CVD risk factors, reduces the possibility of residual confounding in our study, as it is less likely that unmeasured factors might be strongly associated with both alcohol consumption and LV function and unrelated to the covariates we included.

Many studies in alcohol research may be affected by the sick-quitter bias. ${ }^{1415} 17$ Typically, when former drinking is assessed, it is done so during the baseline measurements and hence may be prone to recall bias. Only one study that examined the association between alcohol consumption and LV function ${ }^{23}$ used longitudinal assessment on alcohol consumption, but their results were inconclusive. ${ }^{5}$ In our study, alcohol consumption was also assessed 10 years prior to baseline as part of the HUNT2 study, offering a prospective assessment of former drinking. Notably, excluding former drinkers did not change our results.

The main limitation of our study relates to its cross-sectional design. Second, similar to many previous studies in this field, alcohol consumption was self-reported. Study participants, particularly high consumers, may tend to under-report their alcohol intake, ${ }^{40}{ }^{41}$ although it is unlikely that the rank ordering would be markedly influenced. However, alcohol consumption was weakly correlated with high-density lipoprotein cholesterol level in the HUNT3 population. As less than 1\% of the participants reported consuming more than seven alcoholic drinks per week, we were not able to examine the possible harmful effect of excessive alcohol intake on LV function over this amount of alcohol consumption. Nonetheless, the relatively low level of alcohol intake reported here does also limit our ability to separately assess other dimensions of drinking, such as beverage type and drinking frequency versus quantity, and it allowed us to examine the specific role of binge drinking in an otherwise light-drinking population.

\section{CONCLUSION}

In summary, moderate alcohol consumption was not associated with LV function indices among middle-aged healthy individuals. However, alcohol consumption was positively associated with LV mass among otherwise light consumers, especially among those who report risky drinking behaviours, which highlights the importance of drinking pattern in understanding the cardiovascular effects of alcohol consumption.

\section{Author affiliations}

${ }^{1}$ Department of Public Health Sciences, Karolinska Institutet, Stockholm, Sweden ${ }^{2}$ Department of Public Health and General Practice, Faculty of Medicine, Norwegian University of Science and Technology, Trondheim, Norway

${ }^{3}$ Regional Center for Health Care Improvement, St. Olav's Hospital, Trondheim,

Norway

${ }^{4}$ Department of Circulation and Medical Imaging, Norwegian University of Science and Technology, Trondheim, Norway

${ }^{5}$ Clinic of Cardiology, St. Olavs University Hospital, Trondheim, Norway

${ }^{6}$ Levanger Hospital, Nord-Trøndelag Hospital Trust, Levanger, Norway

${ }^{7}$ Department of Medicine, Beth Israel Deaconess Medical Center, Boston,

Massachusetts, USA

Acknowledgements The Nord-Trøndelag Health Study is a collaborative effort between the Faulty of Medicine, Norwegian University of Science and Technology, Nord-Trøndelag County Council and the Norwegian Institute of Public Health. All laboratory analyses were performed and financed by the Health Trust of NordTrøndelag. We want to thank clinicians and other employees at Nord-Trøndelag Hospital Trust for their support and for contributing to data collection in this research project. 
Contributors All authors fulfill all four authorship criteria. KG, IJ, KDM and HD contributed to conception and design, and all authors contributed to the analyses or the interpretation of the results. KG drafted the manuscript, and IJ, KDL, SA, LBS, LJV and KJM critically revised it. All authors gave final approval and agreed to be accountable for the work.

Funding The study was supported by a KID grant from Karolinska Institutet. Competing interests None declared.

Patient consent Obatined.

Ethics approval the Regional Committee for Medical Research Ethics (REK 4.2009.397)

Provenance and peer review Not commissioned; externally peer reviewed.

Data sharing statement № additional data are available. The authors have no permission for sharing the data.

Open Access This is an Open Access article distributed in accordance with the Creative Commons Attribution Non Commercial (CC BY-NC 4.0) license, which permits others to distribute, remix, adapt, build upon this work non-commercially, and license their derivative works on different terms, provided the original work is properly cited and the use is non-commercial. See: http://creativecommons.org/ licenses/by-nc/4.0/

( $)$ Article author(s) (or their employer(s) unless otherwise stated in the text of the article) 2018. All rights reserved. No commercial use is permitted unless otherwise expressly granted.

\section{REFERENCES}

1. Rehm J, Hasan OSM, Imtiaz S, et al. Quantifying the contribution of alcohol to cardiomyopathy: a systematic review. Alcohol 2017;61:9-15.

2. Iacovoni A, De Maria R, Gavazzi A. Alcoholic cardiomyopathy. J Cardiovasc Med 2010;11:884-92.

3. Larsson SC, Orsini N, Wolk A. Alcohol consumption and risk of heart failure: a dose-response meta-analysis of prospective studies. Eur $J$ Heart Fail 2015;17:367-73.

4. Yousaf H, Rodeheffer RJ, Paterick TE, et al. Association between alcohol consumption and systolic ventricular function: a populationbased study. Am Heart J 2014;167:861-8.

5. Goncalves A, Jhund PS, Claggett B, et al. Relationship between alcohol consumption and cardiac structure and function in the elderly: the atherosclerosis risk in communities study. Circulation 2015;8:e002846.

6. Hung CL, Gonçalves A, Lai YJ, et al. Light to Moderate Habitual Alcohol Consumption Is Associated with Subclinical Ventricular and Left Atrial Mechanical Dysfunction in an Asymptomatic Population: Dose-Response and Propensity Analysis. J Am Soc Echocardiogr 2016;29:1043-51.

7. Manolio TA, Levy D, Garrison RJ, et al. Relation of alcohol intake to left ventricular mass: The Framingham Study. J Am Coll Cardiol 1991;17:717-21.

8. Reisner SA, Lysyansky P, Agmon Y, et al. Global longitudinal strain: a novel index of left ventricular systolic function. J Am Soc Echocardiogr 2004;17:630-3.

9. Mor-Avi V, Lang RM, Badano LP, et al. Current and evolving echocardiographic techniques for the quantitative evaluation of cardiac mechanics: ASE/EAE consensus statement on methodology and indications endorsed by the Japanese Society of Echocardiography. Eur J Echocardiogr 2011;12:167-205.

10. $\mathrm{Ng} \mathrm{ACT,} \mathrm{Prihadi} \mathrm{EA,} \mathrm{Antoni} \mathrm{ML,} \mathrm{et} \mathrm{al.} \mathrm{Left} \mathrm{ventricular} \mathrm{global}$ longitudinal strain is predictive of all-cause mortality independent of aortic stenosis severity and ejection fraction. Eur Heart J Cardiovasc Imaging 2017.

11. Biering-Sørensen T, Biering-Sørensen SR, Olsen FJ, et al. Global Longitudinal Strain by Echocardiography predicts long-term risk of cardiovascular morbidity and mortality in a low-risk general population: the Copenhagen City Heart Study. Circ Cardiovasc Imaging 2017;10:e005521.

12. Dalen $\mathrm{H}$, Thorstensen A, Aase SA, et al. Segmental and global longitudinal strain and strain rate based on echocardiography of 1266 healthy individuals: the HUNT study in Norway. Eur $J$ Echocardiogr 2010;11:176-83.

13. Dalen H, Thorstensen A, Romundstad PR, et al. Cardiovascular risk factors and systolic and diastolic cardiac function: a tissue Doppler and speckle tracking echocardiographic study. J Am Soc Echocardiogr 2011;24:322-32.
14. Shaper AG, Wannamethee G, Walker M. Alcohol and mortality in British men: explaining the U-shaped curve. Lancet 1988;2:1267-73.

15. Klatsky AL, Armstrong MA, Friedman GD. Risk of cardiovascular mortality in alcohol drinkers, ex-drinkers and nondrinkers. Am J Cardiol 1990;66:1237-42.

16. Roerecke M, Rehm J. Alcohol intake revisited: risks and benefits. Curr Atheroscler Rep 2012;14:556-62.

17. Roerecke M, Rehm J. Ischemic heart disease mortality and morbidity rates in former drinkers: a meta-analysis. Am J Epidemiol 2011;173:245-58.

18. Dalen H, Thorstensen A, Vatten LJ, et al. Reference values and distribution of conventional echocardiographic Doppler measures and longitudinal tissue Doppler velocities in a population free from cardiovascular disease. Circ Cardiovasc Imaging 2010;3:614-22.

19. Krokstad S, Langhammer A, Hveem K, et al. Cohort Profile: the HUNT Study, Norway. Int J Epidemiol 2013;42:968-77.

20. Skogen JC, Overland S, Knudsen AK, et al. Concurrent validity of the CAGE questionnaire. The Nord-Trøndelag Health Study. Addict Behav 2011;36:302-7.

21. Skogen JC, Bøe T, Knudsen AK, et al. Psychometric properties and concurrent validity of the CRAFFT among Norwegian adolescents. Ung@hordaland, a population-based study. Addict Behav 2013;38:2500-5.

22. Lang RM, Badano LP, Mor-Avi V, et al. Recommendations for cardiac chamber quantification by echocardiography in adults: an update from the American Society of Echocardiography and the European Association of Cardiovascular Imaging. Eur Heart $J$ Cardiovasc Imaging 2015;16:233-71.

23. Mogelvang R, Biering-Sørensen T, Jensen JS. Tissue Doppler echocardiography predicts acute myocardial infarction, heart failure, and cardiovascular death in the general population. Eur Heart $J$ Cardiovasc Imaging 2015;16:jev180-7.

24. Nes BM, Vatten LJ, Nauman J, et al. A simple nonexercise model of cardiorespiratory fitness predicts long-term mortality. Med Sci Sports Exerc 2014;46:1159-65.

25. Echocardiographic Normal Ranges Meta-Analysis of the Left heart (EchoNoRMAL) Collaboration. A meta-analysis of echocardiographic measurements of the left heart for the development of normative reference ranges in a large international cohort: the EchoNoRMAL study. Eur Heart J Cardiovasc Imaging 2014;15:341-8.

26. Kupari M, Koskinen P. Relation of left ventricular function to habitual alcohol consumption. Am J Cardiol 1993;72:1418-24.

27. Thorstensen A, Dalen $\mathrm{H}$, Amundsen $\mathrm{BH}$, et al. Peak systolic velocity indices are more sensitive than end-systolic indices in detecting contraction changes assessed by echocardiography in young healthy humans. Eur J Echocardiogr 2011;12:924-30.

28. Tsao CW, Gona PN, Salton CJ, et al. Left ventricular structure and risk of cardiovascular events: a framingham heart study cardiac magnetic resonance study. J Am Heart Assoc 2015;4:e002188.

29. Long MJ, Jiang CQ, Lam TH, et al. Alcohol consumption and electrocardiographic left ventricular hypertrophy and mediation by elevated blood pressure in older Chinese men: the Guangzhou Biobank cohort study. Alcohol 2013;47:473-80.

30. Guo R, Ren J. Alcohol dehydrogenase accentuates ethanol-induced myocardial dysfunction and mitochondrial damage in mice: role of mitochondrial death pathway. PLoS One 2010;5:e8757.

31. Matyas C, Varga ZV, Mukhopadhyay P, et al. Chronic plus binge ethanol feeding induces myocardial oxidative stress, mitochondrial and cardiovascular dysfunction, and steatosis. Am J Physiol Heart Circ Physiol 2016;310:H1658-H1670.

32. Bagnardi V, Zatonski W, Scotti L, et al. Does drinking pattern modify the effect of alcohol on the risk of coronary heart disease? Evidence from a meta-analysis. J Epidemiol Community Health 2008;62:615-9.

33. Mukamal KJ, Jensen MK, Grønbaek M, et al. Drinking frequency, mediating biomarkers, and risk of myocardial infarction in women and men. Circulation 2005;112:1406-13.

34. Movva R, Figueredo VM. Alcohol and the heart: to abstain or not to abstain? Int J Cardiol 2013;164:267-76.

35. Zagrosek A, Messroghli D, Schulz O, et al. Effect of binge drinking on the heart as assessed by cardiac magnetic resonance imaging. JAMA 2010;304:1328-30.

36. Voskoboinik A, Prabhu S, Ling LH, et al. Alcohol and atrial fibrillation: a sobering review. J Am Coll Cardiol 2016;68:2567-76.

37. Sagli J. Norse drikkekulturer Geografi, sosial bakgrunn,livsstil og tilgjengelig (Norwegian drinking culture: geography, social background, lifestyle and availability). Report of the Norwegian Institute of Alcohol and Drug Research. Oslo, Norway: Norwegian Institute of Alcohol and Drug Research, 1994. 
38. Lucas N, Windsor TD, Caldwell TM, et al. Psychological distress in non-drinkers: associations with previous heavy drinking and current social relationships. Alcohol Alcohol 2010;45:95-102.

39. Boden JM, Fergusson DM. Alcohol and depression. Addiction 2011;106:906-14.
40. Høyer G, Nilssen O, Brenn T, et al. Påliteligheten av selvrapportert alkoholkonsum. Svalbardstudien 1988-89. Norsk Epidemiologi 1996;6:109-13.

41. Midanik $L$. The validity of self-reported alcohol consumption and alcohol problems: a literature review. Br J Addict 1982;77:357-82. 